\title{
Maternal and neonatal factors associated with mode of delivery under a universal newborn hearing screening programme in Lagos, Nigeria
}

\author{
Bolajoko O Olusanya*1 and Olumuyiwa A Solanke ${ }^{2}$
}

\author{
Address: ${ }^{1}$ Maternal and Child Health Unit, Department of Community Health and Primary Care College of Medicine, University of Lagos, \\ Surulere, Lagos, Nigeria and ${ }^{2}$ Lagos Island Maternity Hospital, Catholic Mission Street, Lagos, Nigeria \\ Email: Bolajoko O Olusanya* - boolusanya@aol.com; Olumuyiwa A Solanke - labamba122@yahoo.com \\ * Corresponding author
}

Published: 4 September 2009

BMC Pregnancy and Childbirth 2009, 9:4I doi:|0.1|86/I47|-2393-9-4|

This article is available from: http://www.biomedcentral.com/l47|-2393/9/4

(c) 2009 Olusanya and Solanke; licensee BioMed Central Ltd.

This is an Open Access article distributed under the terms of the Creative Commons Attribution License (http://creativecommons.org/licenses/by/2.0), which permits unrestricted use, distribution, and reproduction in any medium, provided the original work is properly cited.

\begin{abstract}
Background: Emerging evidence from a recent pilot universal newborn hearing screening (UNHS) programme suggests that the burden of obstetric complications associated with mode of delivery is not limited to maternal and perinatal mortality but may also include outcomes that undermine optimal early childhood development of the surviving newborns. However, the potential pathways for this association have not been reported particularly in the context of a resource-poor setting. This study therefore set out to establish the pattern of delivery and the associated neonatal outcomes under a UNHS programme.
\end{abstract}

Methods: A cross-sectional study in which all consenting mothers who delivered in an inner-city tertiary maternity hospital in Lagos, Nigeria from May 2005 to December 2007 were enrolled during the UNHS programme. Socio-demographic, obstetric and neonatal factors independently associated with vaginal, elective and emergency caesarean deliveries were determined using multinomial logistic regression analyses.

Results: Of the 4615 mothers enrolled, 2584 (56.0\%) deliveries were vaginal, 1590 (34.4\%) emergency caesarean and $44 \mathrm{I}(9.6 \%)$ elective caesarean section. Maternal age, parity, social class and all obstetric factors including lack of antenatal care, maternal HIV and multiple gestations were associated with increased risk of emergency caesarean delivery compared with vaginal delivery. Only parity, lack of antenatal care and prolonged/obstructed labour were associated with increased risk of emergency compared with elective caesarean delivery. Infants delivered by vaginal method or by emergency caesarean section were more likely to be associated with the risk of sensorineural hearing loss but less likely to be associated with hyperbilirubinaemia compared with infants delivered by elective caesarean section. Emergency caesarean delivery was also associated with male gender, low five-minute Apgar scores and admission into special care baby unit compared with vaginal or elective caesarean delivery.

Conclusions: The vast majority of caesarean delivery in this population occur as emergencies and are associated with socio-demographic factors as well as several obstetric complications. Mode of delivery is also associated with the risk of sensorineural hearing loss and other adverse birth outcomes that lie on the causal pathways for potential developmental deficits. 


\section{Background}

It is now widely acknowledged that effective efforts aimed at improving child health in resource-poor countries must be preceded and underpinned by improvement in maternal health within a continuum of care from pregnancy to adolescence [1]. This is corroborated by substantial evidence showing that regions such as sub-Saharan Africa and South Asia with the highest rates of maternal mortality also have the highest burden of infant and child mortality worldwide [2-5]. Maternal deaths in these regions are predominantly attributable to obstetric complications during pregnancy and childbirth [3], and can be averted or substantially curtailed through availability and access/ proximity to modern obstetric services by skilled attendants [6]. In many developing countries, such services are found mostly in urban areas, but they are increasingly being directed to rural areas under various global health initiatives [7].

In many developing countries undue delays in initiating life-saving surgical intervention for women at risk of severe complications among other factors may undermine the envisaged outcomes from facility-based services $[3,8]$. Such delays may be due to delay in seeking essential obstetric care; in reaching the hospital or appropriate health facility; or in receiving adequate care in the hospital [8]. For example, barring the absence of other barriers such as cost and accessibility, refusal of life-saving caesarean section is not uncommon among women in urban settings in sub-Saharan Africa particularly in a country like Nigeria which is a leading contributor to regional and global burden of maternal mortality [9-13].

Typically, in secondary and tertiary maternity hospitals many mothers arrive in a moribund state or with complications that may either lead to death or severe morbidity and disability in the mother after emergency surgical intervention $[3,14,15]$. While perinatal mortality associated with complications during pregnancy and childbirth has been extensively reported in the literature, evidence linking mode of delivery (with or without obstetric complications) to the risk of developmental disabilities in the surviving newborns in developing countries is rare [16].

Emerging evidence from a recently concluded pilot universal newborn hearing screening (UNHS) programme in a maternity hospital in Nigeria for example suggests that mode of delivery may be associated with sensorineural hearing loss in the surviving newborns [17]. This is consistent with existing reports associating the greatest burden of developmental disabilities worldwide with countries such as Nigeria, India and Pakistan besides having the highest rates of maternal and child mortality [18]. However, the potential pathways for this association have not been reported. We hypothesised that adverse neonatal outcomes commonly associated with mode of delivery in a resource-poor country are directly associated with or lie on the causal pathways of developmental disabilities such as sensorineural hearing loss.

This study therefore set out to establish the pattern of delivery and the associated maternal factors; determine infant factors/neonatal outcomes associated with mode of delivery; as well as identify possible direct or indirect links between mode of delivery and developmental deficits in early infancy under a UNHS programme in a developing country.

\section{Methods}

\section{Study design and population}

This cross-sectional study was conducted in an inner-city tertiary maternity hospital in Lagos, Nigeria from May 2005 to December 2007. Lagos is the most populous city in sub-Saharan Africa and the hospital is the oldest maternity hospital in metropolitan Lagos providing specialist services to several private and public hospitals within and outside its catchment area. The hospital is owned and managed by the state government as a public health institution. All live births over the study period were eligible for enrolment into the study, excluding those who did not survive 24 hours after delivery. Newborns whose mothers were too ill to be interviewed including near-misses and those who died during childbirth were also excluded. Ethical approvals were obtained from Lagos State Health Management Board, Nigeria and University College London, UK as part of a wider UNHS pilot project [17].

\section{Main outcome variables}

Three modes of delivery were considered as outcome measures namely: vaginal delivery, elective and emergency caesarean section. Vaginal delivery included spontaneous vertex and assisted/instrumental delivery such as breech, forceps and vacuum. Caesarean section was termed elective if the decision for the operation was made before onset of labour because the pregnancy was considered to be high-risk and/or mother was referred from antenatal clinic. Elective caesarean section in this population was rarely based on non-medical reasons. Emergency caesarean section referred to operations prompted by a diagnosis of fetal distress, vaginal bleeding, premature rupture of membrane, antepartum haemorrhage or hypertensive conditions. The term also embraced emergency intrapartum caesarean section initiated during labour.

\section{Maternal factors}

The factors of interest were guided by evidence from published literature [19-21] and the available data from hospital records of the participants. These included sociodemographic factors such as age, marital status, parity, ethnicity, religion, education, occupation, social class, type of residential accommodation (rented or owned) and sanitation facilities (shared or self-contained). Social 
classes were determined based on mother's education and father's occupation [22]. Social class I was termed as "high", II or III as "middle" and IV or V as "low". Maternal factors also included variables reflecting health-seeking behaviours such as antenatal care and traditional herbal drug use in pregnancy; obstetric indications for caesarean delivery such as hypertensive conditions (including preeclampsia, eclampsia and pregnancy induced hypertension), antepartum haemorrhage, cephalopelvic disproportion, premature rupture of membranes, prolonged and/or obstructed labour, malpresentation, previous caesarean delivery, fetal distress and other obstetric conditions such as maternal HIV status and multiple pregnancies.

\section{Infant factors/outcomes}

The neonatal factors or outcomes of interest were infant's gender, gestational age, birthweight, Apgar scores at one minute and five minutes, hyperbilirubinaemia, admission into special care baby unit (SCBU) and hearing screening outcomes. SCBU admission is a helpful surrogate for a range of adverse perinatal conditions that cannot be readily ascertained in hospitals with limited diagnostic facilities. Hearing screening outcomes were based on a twostage hearing screening protocol consisting of a first-stage screening with transient evoked otoacoustic emissions followed by a second-stage of automated auditory brainstem response test as previously reported [17]. Maternal and neonatal mortality associated with mode of delivery were not considered in this study due to incomplete data for the entire period of this study.

\section{Statistical Analysis}

Cross-tabulation of the outcome and explanatory variables was done to provide a descriptive overview of our study population. Multinomial logistic regression model was used as it is an appropriate modelling tool where there are more than two discrete outcomes. It estimates the effect of the independent variables on the probability of a particular method of delivery. The three categories of delivery (vaginal, elective and emergency caesarean section) as specified in this study are sufficiently distinct to satisfy the assumption of independent alternatives. Unconditional univariable multinomial logistic regression analysis was first performed for each independent variable against the dependent variable (mode of delivery) to examine the unadjusted association with the three modes of delivery. The strength of association was estimated by odds ratios (OR) and the corresponding 95\% confidence intervals (CI). All biologically plausible factors and those with significance $(\mathrm{p}<0.05)$ or borderline significance $(0.10>\mathrm{p} \geq 0.05)$ were entered into the multinomial multivariable logistic model to assess the effect of each variable independently on the mode of delivery while controlling for the potential confounding effects of cov- ariates. There were no a priori hypotheses for interaction terms so these were not investigated. Finally, neonatal factors or outcomes significantly associated with mode of delivery were determined after adjusting for all sociodemographic and obstetric factors. Model performance was estimated with the Nagelkerke Pseudo- $R^{2}$ statistic (a measure of explained variation in the model). Missing data were managed by exclusion in all of the analyses. All statistical analyses were done with SPSS Windows version 16.0 (SPSS Inc, Chicago, IL, USA).

\section{Results}

A total of $4615(81.9 \%)$ consenting mothers with live births out of 5636 recorded deliveries (including perinatal deaths) at the hospital over the study period were enrolled. In all, 2584 (56.0\%) mothers had vaginal delivery, 1590 (34.4\%) emergency caesarean section and 441 (9.6\%) elective caesarean section. Majority of the mothers were between the ages of 20-35 years, married, of the Yoruba ethnic group, had a minimum of secondary education, belonged to the middle social class and lived in rented accommodation with almost half (48.2\%) having shared sanitation facilities (Table 1). Emergency caesarean section rate was highest among Yoruba tribe $(69.6 \%)$, Christian mothers (65.1\%), those living in rented accommodation (96\%) and those in the middle social class $(73.2 \%)$. More than half $(54.2 \%)$ of mothers who had emergency caesarean section were primiparous. The obstetric profile of the mothers showed that about a third $(34.9 \%)$ did not attend antenatal clinics for their current delivery and almost one-fifth (19.1\%) reported using herbal medication during pregnancy (Table 2). A total of 1544 (33.5\%) from the total study population had at least one medical indication for caesarean section and 1352 $(87.6 \%)$ of this group had caesarean section. Conversely, caesarean section was medically indicated in $66.6 \%$ of all those who had such intervention. Previous caesarean section and prolonged/obstructed labour were the commonest maternal obstetric indications in the total population. Emergency caesarean section rate was highest among mothers who did not attend antenatal clinics (53.1\%). About $6 \%$ of the mothers were diagnosed with HIV.

The univariate multinomial analyses showed that apart from marital status and religion all socio-demographic and obstetric factors were significantly associated with mode of delivery. Nonetheless, all factors were entered into the multivariable logistic regression models in deriving the final prediction models. While the model consisting only of the socio-demographic factors (Table 3) explained only $6 \%$ (Nagelkerke $R^{2}=0.058$ ) of the variation in mode of delivery, the model for obstetric factors (Table 4) explained about 52\% (Nagelkerke $R^{2}=0.517$ ). The addition of socio-demographic factors marginally increased the explained variations in the model for obstet- 
Table I: Socio-demographic factors of mothers by mode of delivery

\begin{tabular}{|c|c|c|c|c|}
\hline Factors & $\begin{array}{l}\text { Total deliveries (\%) } \\
\mathrm{n}=46 \mathrm{I5}\end{array}$ & $\begin{array}{l}\text { Vaginal delivery (\%) } \\
n=2584\end{array}$ & $\begin{array}{l}\text { Emergency caesarean } \\
\text { section (\%) } \\
n=1590\end{array}$ & $\begin{array}{l}\text { Elective caesarean } \\
\text { section (\%) } \\
n=44 I\end{array}$ \\
\hline \multicolumn{5}{|l|}{ Age (Years) [a] } \\
\hline$<20$ & $77(1.7)$ & $41(1.6)$ & $32(2.0)$ & $4(0.9)$ \\
\hline $20-35$ & $4011(87.1)$ & $2275(88.1)$ & $1379(87.1)$ & $357(81.0)$ \\
\hline$>35$ & $519(11.3)$ & $266(10.3)$ & $173(10.9)$ & $80(18.1)$ \\
\hline \multicolumn{5}{|l|}{ Marital status } \\
\hline Married & 4519 (97.9) & $2530(97.9)$ & I555 (97.8) & $434(98.4)$ \\
\hline Not married & $96(2.1)$ & $54(2.1)$ & $35(2.2)$ & $7(1.6)$ \\
\hline \multicolumn{5}{|l|}{ Parity } \\
\hline Primiparous & $2316(50.2)$ & $1290(49.9)$ & $86 \mid(54.2)$ & $165(37.4)$ \\
\hline Multiparous & $2299(49.8)$ & $1294(50.1)$ & $729(45.8)$ & $276(62.6)$ \\
\hline \multicolumn{5}{|l|}{ Ethnicity } \\
\hline Yoruba & $3478(75.4)$ & 2055 (79.5) & $1107(69.6)$ & 316 (7I.7) \\
\hline Hausa & $128(2.8)$ & $77(3.0)$ & $43(2.7)$ & $8(1.8)$ \\
\hline Ibo \& Others & $1009(21.9)$ & $452(17.5)$ & $440(27.7)$ & $117(26.5)$ \\
\hline \multicolumn{5}{|l|}{ Religion } \\
\hline Muslim & I 888 (40.9) & $1173(45.4)$ & $555(34.9)$ & $160(36.3)$ \\
\hline Christianity & $2727(59.1)$ & $|4| \mid(54.6)$ & $1035(65.1)$ & $28 I(63.7)$ \\
\hline \multicolumn{5}{|l|}{ Education } \\
\hline Primary or none & $420(9.1)$ & $244(9.5)$ & $147(9.3)$ & $29(6.5)$ \\
\hline Secondary & $2419(52.4)$ & I $344(52.0)$ & $872(54.8)$ & $203(46.0)$ \\
\hline Tertiary & $1776(38.5)$ & $996(38.5)$ & $57 \mid(35.9)$ & $209(47.4)$ \\
\hline \multicolumn{5}{|l|}{ Occupation } \\
\hline None & $899(19.5)$ & $534(20.7)$ & $309(19.4)$ & $56(12.7)$ \\
\hline Small trade/casual job & $2309(50)$ & $1296(43.3)$ & $803(50.5)$ & $210(47.7)$ \\
\hline Full-time job & $1407(30.5)$ & $754(29.2)$ & $478(30.1)$ & 175 (39.7) \\
\hline \multicolumn{5}{|l|}{ Social class } \\
\hline High & $808(17.5)$ & $44 I(17.1)$ & $244(15.3)$ & $123(27.9)$ \\
\hline Middle & $3231(70.0)$ & I79| (69.3) & $1164(73.2)$ & $276(62.6)$ \\
\hline Low & $576(12.5)$ & $352(13.6)$ & $182(11.4)$ & $42(9.5)$ \\
\hline \multicolumn{5}{|l|}{ Accommodation } \\
\hline Owned & $228(4.9)$ & $150(5.8)$ & $63(4.0)$ & $15(3.4)$ \\
\hline Rented & $4387(95.1)$ & $2434(94.2)$ & $1527(96.0)$ & $426(96.6)$ \\
\hline \multicolumn{5}{|l|}{ Housing sanitation facilities } \\
\hline Not Shared & $2391(51.8)$ & $132 \mid(5 \mid .1)$ & $792(49.8)$ & $278(63.0)$ \\
\hline Shared & 2224 (48.2) & $1263(48.9)$ & $798(50.2)$ & $163(37.0)$ \\
\hline
\end{tabular}

Missing data: $[\mathrm{a}]=8(0.2 \%)$.

ric factors by $1 \%$ to about $53 \%$ (Nagelkerke $R^{2}=0.534$ ). No evidence of any collinearity among the factors was found in the final models.

\section{Emergency caesarean section compared with vaginal delivery}

Older mothers, those who were first-time mothers or in the middle social class had increased odds of emergency caesarean section although 24\% lower odds were observed among Yoruba mothers. As expected, all the obstetric factors increased the odds of emergency caesarean section with antepartum haemorrhage, cephalopelvic disproportion, prolonged/obstructed labour, previous caesarean section and fetal distress showing the highest odds. Although maternal HIV was associated with $40 \%$ increased odds for emergency caesarean section, the dif- 
Table 2: Obstetric factors by mode of delivery

\begin{tabular}{|c|c|c|c|c|}
\hline Maternal profile & $\begin{array}{l}\text { Total deliveries (\%) } \\
n=4615\end{array}$ & $\begin{array}{l}\text { Vaginal delivery (\%) } \\
n=2584\end{array}$ & $\begin{array}{l}\text { Emergency caesarean } \\
\text { section }(\%) \\
n=1590\end{array}$ & $\begin{array}{l}\text { Elective caesarean } \\
\text { section }(\%) \\
n=44 I\end{array}$ \\
\hline \multicolumn{5}{|l|}{ Antenatal care } \\
\hline $\begin{array}{l}\text { One or more visits } \\
\text { None }\end{array}$ & $\begin{array}{l}3006(65.1) \\
1609(34.9)\end{array}$ & $\begin{array}{l}1910(73.9) \\
674(26.1)\end{array}$ & $\begin{array}{l}745(46.9) \\
845(53.1)\end{array}$ & $\begin{array}{l}351(79.6) \\
90(20.4)\end{array}$ \\
\hline \multicolumn{5}{|l|}{ Herbal drug in pregnancy } \\
\hline $\begin{array}{l}\text { None } \\
\text { Yes }\end{array}$ & $\begin{array}{l}3732(80.9) \\
883(19.1)\end{array}$ & $\begin{array}{l}2020(78.2) \\
564(21.8)\end{array}$ & $\begin{array}{l}1329(83.6) \\
26 \mid(16.4)\end{array}$ & $\begin{array}{l}383(86.8) \\
58(13.2)\end{array}$ \\
\hline \multicolumn{5}{|l|}{ Hypertensive conditions } \\
\hline $\begin{array}{l}\text { No } \\
\text { Yes }\end{array}$ & $\begin{array}{l}4317(93.5) \\
298(6.5)\end{array}$ & $\begin{array}{l}2499(96.7) \\
85(3.3)\end{array}$ & $\begin{array}{l}1415(89.0) \\
175(11.0)\end{array}$ & $\begin{array}{l}403(91.4) \\
38(8.6)\end{array}$ \\
\hline \multicolumn{5}{|c|}{ Ante-partum haemorrhage } \\
\hline $\begin{array}{l}\text { No } \\
\text { Yes }\end{array}$ & $\begin{array}{l}4566(98.9) \\
49(1.1)\end{array}$ & $\begin{array}{l}2582(99.9) \\
2(0.1)\end{array}$ & $\begin{array}{l}1546(97.2) \\
44(2.8)\end{array}$ & $\begin{array}{l}438(99.3) \\
3(0.7)\end{array}$ \\
\hline \multicolumn{5}{|c|}{ Cephalopelvic disproportion } \\
\hline $\begin{array}{l}\text { No } \\
\text { Yes }\end{array}$ & $\begin{array}{l}4433(96.1) \\
182(3.9)\end{array}$ & $\begin{array}{l}2580(99.8) \\
4(0.2)\end{array}$ & $\begin{array}{l}\text { I } 443(90.8) \\
\text { | } 47(9.2)\end{array}$ & $\begin{array}{l}410(93.0) \\
31(7.0)\end{array}$ \\
\hline \multicolumn{5}{|l|}{ Premature rupture of } \\
\hline $\begin{array}{l}\text { No } \\
\text { Yes }\end{array}$ & $\begin{array}{l}4565(98.9) \\
50(1.1)\end{array}$ & $\begin{array}{l}2568(99.4) \\
16(0.6)\end{array}$ & $\begin{array}{l}156 \mid(98.2) \\
29(1.8)\end{array}$ & $\begin{array}{l}436(98.9) \\
5(1.1)\end{array}$ \\
\hline \multicolumn{5}{|c|}{ Prolonged/obstructed labour } \\
\hline $\begin{array}{l}\text { No } \\
\text { Yes }\end{array}$ & $\begin{array}{l}4190(90.8) \\
425(9.2)\end{array}$ & $\begin{array}{l}2565(99.3) \\
19(0.7)\end{array}$ & $\begin{array}{l}1205(75.8) \\
385(24.2)\end{array}$ & $\begin{array}{l}420(95.2) \\
2 I^{*}(4.8)\end{array}$ \\
\hline \multicolumn{5}{|l|}{ Malpresentation } \\
\hline $\begin{array}{l}\text { No } \\
\text { Yes }\end{array}$ & $\begin{array}{l}4338(94.0) \\
277(6.0)\end{array}$ & $\begin{array}{l}2521(97.6) \\
63(2.4)\end{array}$ & $\begin{array}{l}1425(89.6) \\
165(10.4)\end{array}$ & $\begin{array}{l}392(89.9) \\
49(11.1)\end{array}$ \\
\hline \multicolumn{5}{|c|}{ Previous caesarean section } \\
\hline $\begin{array}{l}\text { No } \\
\text { Yes }\end{array}$ & $\begin{array}{l}4152(90.0) \\
463(10.0)\end{array}$ & $\begin{array}{l}2569(99.4) \\
15(0.6)\end{array}$ & $\begin{array}{l}1325(83.3) \\
265(16.7)\end{array}$ & $\begin{array}{l}258(58.5) \\
183(41.5)\end{array}$ \\
\hline \multicolumn{5}{|l|}{ Maternal HIV } \\
\hline $\begin{array}{l}\text { No } \\
\text { Yes }\end{array}$ & $\begin{array}{l}4350(94.3) \\
265(5.7)\end{array}$ & $\begin{array}{l}244 \mid(94.5) \\
\mid 43(5.5)\end{array}$ & $\begin{array}{l}1507(94.8) \\
83(5.2)\end{array}$ & $\begin{array}{l}402(91.2) \\
39(8.8)\end{array}$ \\
\hline \multicolumn{5}{|l|}{ Multiple gestations } \\
\hline $\begin{array}{l}\text { No } \\
\text { Yes }\end{array}$ & $\begin{array}{l}43 I 4(93.5) \\
30 I(6.5)\end{array}$ & $\begin{array}{l}2454(95.0) \\
130(5.0)\end{array}$ & $\begin{array}{l}1458(91.7) \\
132(8.3)\end{array}$ & $\begin{array}{l}402(91.2) \\
39(8.8)\end{array}$ \\
\hline \multicolumn{5}{|l|}{ Fetal distress } \\
\hline $\begin{array}{l}\text { No } \\
\text { Yes }\end{array}$ & $\begin{array}{l}4485(97.2) \\
130(2.8)\end{array}$ & $\begin{array}{l}2581(99.9) \\
3(0.1)\end{array}$ & $\begin{array}{l}\text { I } 465(92.1) \\
\text { I } 25(7.9)\end{array}$ & $\begin{array}{l}439(99.5) \\
2(0.5)\end{array}$ \\
\hline
\end{tabular}

*Possible error in patient records due to prior consent for elective caesarean section at booking. However no material effect was observed when reflected in the analysis

ference was only marginally significant ( $p=0.054)$. Mothers who used herbal medications in pregnancy were found to have $24 \%$ lower odds for emergency caesarean section.

\section{Emergency caesarean section compared with elective caesarean section}

Maternal age and occupation were the only socio-demographic factors predictive of emergency caesarean section among those who required surgical intervention. Fetal distress was associated with the largest odds for emer- gency caesarean section while HIV-positive status and previous caesarean section were associated with over $50 \%$ decreased odds of emergency caesarean section. Hypertensive disorders, cephalopelvic disproportion, premature rupture of membranes and malpresentation were not discriminatory among mothers who had caesarean section.

Vaginal delivery compared with elective caesarean section Compared to women in the active childbearing age (20 35 years), women older than 35 years had 56\% lower 
Table 3: Socio-demographic predictors of mode of delivery after multinomial logistic regression $\$$

\begin{tabular}{|c|c|c|c|}
\hline Factor & $\begin{array}{l}\text { Emergency c-section vs vaginal } \\
\text { delivery } \\
\text { Adjusted odds ratio } \\
\text { (95\% Confidence interval) }\end{array}$ & $\begin{array}{l}\text { Emergency c-section vs elective } \\
\text { c-section } \\
\text { Adjusted odds ratio } \\
\text { (95\% Confidence interval) }\end{array}$ & $\begin{array}{l}\text { Vaginal delivery vs elective c- } \\
\text { section } \\
\text { Adjusted odds ratio } \\
\text { (95\% Confidence interval) }\end{array}$ \\
\hline \multicolumn{4}{|l|}{ Age (Years) } \\
\hline$<20$ & $1.18(0.62-2.22)$ & $1.09(0.35-3.38)$ & $0.93(0.30-2.86)$ \\
\hline $20-35$ & 1.0 & 1.0 & 1.0 \\
\hline$>35$ & $1.58(1.22-2.06)^{* *}$ & $0.69(0.50-0.95)^{*}$ & $0.44(0.31-0.61)^{* * *}$ \\
\hline \multicolumn{4}{|l|}{ Marital status } \\
\hline Married & $1.17(0.64-2.14)$ & $1.61(0.63-4.10)$ & $1.38(0.54-3.48)$ \\
\hline Not married & 1.0 & 1.0 & 1.0 \\
\hline \multicolumn{4}{|l|}{ Parity } \\
\hline Primiparous & $1.51(1.26-1.81)^{* * *}$ & $1.27(0.98-1.66)$ & $0.84(0.65-1.10)$ \\
\hline Multiparous & 1.0 & 1.0 & 1.0 \\
\hline \multicolumn{4}{|l|}{ Ethnicity } \\
\hline Yoruba & $0.76(0.6 \mathrm{I}-0.95)^{* *}$ & $1.07(0.79-1.45)$ & $1.40(1.03-1.91)^{*}$ \\
\hline Hausa & $0.68(0.40-1.16)$ & $1.40(0.60-3.29)$ & $2.07(0.87-4.91)$ \\
\hline Ibo \& Others & 1.0 & 1.0 & 1.0 \\
\hline \multicolumn{4}{|l|}{ Religion } \\
\hline Muslim & $0.90(0.74-1.09)$ & $0.85(0.64-1.13)$ & $0.95(0.71-1.26)$ \\
\hline Christianity & 1.0 & 1.0 & 1.0 \\
\hline \multicolumn{4}{|l|}{ Education } \\
\hline Primary or none & $1.15(0.78-1.69)$ & $1.27(0.72-2.25)$ & $1.10(0.62-1.97)$ \\
\hline Secondary & $1.08(0.85-1.39)$ & $1.10(0.78-1.57)$ & $1.02(0.7 \mid-1.46)$ \\
\hline Tertiary & 1.0 & 1.0 & 1.0 \\
\hline \multicolumn{4}{|l|}{ Occupation } \\
\hline None & $0.84(0.66-1.08)$ & $1.45(1.00-2.11)^{*}$ & $1.72(1.19-2.50)^{* *}$ \\
\hline Small trade/casual job & $0.95(0.76-1.20)$ & $0.97(0.71-1.34)$ & $\mathrm{I} .02(0.74-\mathrm{I} .4 \mathrm{I})$ \\
\hline Full-time job & 1.0 & 1.0 & 1.0 \\
\hline \multicolumn{4}{|l|}{ Social class } \\
\hline High & $1.28(0.86-1.89)$ & $0.66(0.38-1.13)$ & $0.5 \mathrm{I}(0.30-0.89)^{*}$ \\
\hline Middle & $1.37(1.03-1.83)^{*}$ & $1.22(0.80-1.85)$ & $0.89(0.58-1.35)$ \\
\hline Low & 1.0 & 1.0 & 1.0 \\
\hline \multicolumn{4}{|l|}{ Accommodation } \\
\hline Owned & $0.64(0.43-0.98)^{*}$ & $1.33(0.70-2.50)$ & $2.06(1.09-3.88)^{*}$ \\
\hline Rented & 1.0 & 1.0 & 1.0 \\
\hline \multicolumn{4}{|l|}{ Housing sanitation facilities } \\
\hline Not Shared & $1.14(0.94-1.38)$ & $0.84(0.64-1.11)$ & $0.74(0.56-0.98)^{*}$ \\
\hline Shared & 1.0 & 1.0 & 1.0 \\
\hline
\end{tabular}

\$After adjusting for all obstetric factors; $*_{p}<0.05 ; * * p<0.01$; ${ }^{* *} \mathrm{p}<0.00 \mathrm{I}$. c-section = caesarean section; vs = versus/compared with

odds of vaginal delivery while being a Yoruba woman or having no occupation was associated with increased odds of vaginal delivery. Similarly, living in owned residential accommodation increased the odds of vaginal delivery by two-fold compared to those in rented accommodation while expectedly, living in an apartment without shared sanitation facilities decreased the odds of vaginal delivery by $26 \%$. Almost all the obstetric factors including multi- ple pregnancies but excluding premature rupture of membranes were associated with decreased odds for vaginal delivery. Lack of antenatal care and use of herbal drug in pregnancy were not significantly associated with vaginal delivery.

Overall, marital status, religion and education were not associated with any mode of delivery while maternal age 
Table 4: Obstetric predictors of mode of delivery after multinomial logistic regression $\$$

\begin{tabular}{|c|c|c|c|}
\hline Factor & $\begin{array}{l}\text { Emergency c-section vs } \\
\text { vaginal delivery } \\
\text { Adjusted odds ratio } \\
\text { (95\% Confidence interval) }\end{array}$ & $\begin{array}{l}\text { Emergency c-section vs } \\
\text { elective c-section } \\
\text { Adjusted odds ratio } \\
\text { (95\% Confidence interval) }\end{array}$ & $\begin{array}{l}\text { Vaginal delivery vs } \\
\text { elective c-section } \\
\text { Adjusted odds ratio } \\
\text { (95\% Confidence interval) }\end{array}$ \\
\hline \multicolumn{4}{|l|}{ Antenatal care } \\
\hline One or more visits & 1.0 & 1.0 & 1.0 \\
\hline None & $2.54(2.14-3.03)^{* * *}$ & $3.23(2.46-4.24)^{* * *}$ & $1.27(0.96-1.68)$ \\
\hline \multicolumn{4}{|c|}{ Herbal drug in pregnancy } \\
\hline None & 1.0 & 1.0 & 1.0 \\
\hline Yes & $0.76(0.60-0.95)^{*}$ & $1.06(0.75-1.5 \mathrm{I})$ & $1.40(0.99-1.99)$ \\
\hline \multicolumn{4}{|l|}{ Hypertensive conditions } \\
\hline No & 1.0 & 1.0 & 1.0 \\
\hline Yes & $4.80(3.52-6.52)^{* * *}$ & $1.12(0.75-1.68)$ & $0.23(0.15-0.36)^{* * *}$ \\
\hline \multicolumn{4}{|c|}{ Ante-partum haemorrhage } \\
\hline No & 1.0 & 1.0 & 1.0 \\
\hline Yes & $72.58(17.08-308.47)^{* * *}$ & $3.49(1.04-11.72)^{*}$ & $0.05(0.01-0.30)^{* *}$ \\
\hline \multicolumn{4}{|c|}{ Cephalopelvic disproportion } \\
\hline No & 1.0 & 1.0 & 1.0 \\
\hline Yes & $79.83(29.00-219.78)^{* * * *}$ & $1.36(0.87-2.13)$ & $0.02(0.01-0.05)^{* * *}$ \\
\hline \multicolumn{4}{|c|}{ Premature rupture of membranes } \\
\hline No & 1.0 & 1.0 & 1.0 \\
\hline Yes & $3.38(1.66-6.87)^{* * *}$ & $1.93(0.70-5.31)$ & $0.57(0.19-1.75)$ \\
\hline \multicolumn{4}{|c|}{ Prolonged/obstructed labour } \\
\hline No & 1.0 & 1.0 & 1.0 \\
\hline Yes & $59.95(37.11-96.84)^{* * *}$ & $5.06(3.16-8.10)^{* * *}$ & $0.08(0.04-0.16)^{* * *}$ \\
\hline \multicolumn{4}{|l|}{ Malpresentation } \\
\hline No & 1.0 & 1.0 & 1.0 \\
\hline Yes & $6.70(4.82-9.32)^{* * *}$ & $0.91(0.63-1.32)$ & $0.14(0.09-0.21)^{* * *}$ \\
\hline \multicolumn{4}{|c|}{ Previous caesarean section } \\
\hline No & 1.0 & 1.0 & 1.0 \\
\hline Yes & $59.41(34.46-102.42)^{* * *}$ & $0.41(0.31-0.54)^{* * *}$ & $0.01(0.00-0.01)^{* * *}$ \\
\hline \multicolumn{4}{|l|}{ Maternal HIV } \\
\hline No & 1.0 & 1.0 & 1.0 \\
\hline Yes & $1.40(1.00-1.97)^{*}$ & $0.45(0.29-0.69)^{* * *}$ & $0.32(0.21-0.49)^{* * *}$ \\
\hline \multicolumn{4}{|l|}{ Multiple gestations } \\
\hline No & 1.0 & 1.0 & 1.0 \\
\hline Yes & $2.39(1.75-3.26)^{* * *}$ & $1.08(0.71-1.64)$ & $0.45(0.29-0.70)^{* * *}$ \\
\hline \multicolumn{4}{|l|}{ Fetal distress } \\
\hline No & 1.0 & 1.0 & 1.0 \\
\hline Yes & II $6.25(36.28-372.50)^{* * * *}$ & $|6.6|(4.04-68.29)^{* * *}$ & $0.14(0.02-0.87)^{*}$ \\
\hline
\end{tabular}

\$After adjusting for all socio-demographic factors; ${ }^{*} \mathrm{p}<0.05$; ${ }^{*} \mathrm{p}<0.0 \mathrm{I}$; $* * * \mathrm{p}<0.00 \mathrm{I}$. c-section = caesarean section; vs $=$ versus/compared with 
was a consistent socio-demographic predictor for vaginal delivery or caesarean section.

\section{Neonatal outcomes/factors by mode of delivery}

Neonatal factors associated with mode of delivery after adjusting for all maternal factors are presented in Table 5. Infants delivered by vaginal method or emergency caesarean section were more likely to be associated with the risk of sensorineural hearing loss but less likely to be associated with hyperbilirubinaemia compared with infants delivered by elective caesarean section. Emergency caesarean delivery was also associated with male gender, low five-minute Apgar scores and admission into special care baby unit (SCBU) compared with vaginal or elective caesarean delivery. Infants delivered by emergency caesarean section were less likely to be preterm while those delivered by vaginal method were more likely to have low birthweight compared to infants delivered by elective caesarean section.

\section{Discussion}

The study has shown that the rate of caesarean section in this tertiary maternity hospital is high and the substantial proportion was by emergency surgical intervention. Our study has also shown that emergency caesarean section in this hospital is associated with a wide range of obstetric complications resulting in adverse outcomes for the surviving newborns. These findings need to be set against the backdrop of the fact that the study was conducted in an inner-city community where the vast majority of mothers belonged to the middle or high social class but over half (52\%) still delivered outside hospital facilities despite

Table 5: Neonatal factors associated with mode of delivery after multinomial logistic regression\$

\begin{tabular}{|c|c|c|c|c|}
\hline Factor & $\begin{array}{l}\text { Total (\%) } \\
n=46 \text { I5 }\end{array}$ & $\begin{array}{l}\text { Emergency c-section vs } \\
\text { vaginal delivery } \\
\text { Adjusted odds ratio } \\
\text { (95\% Confidence interval) }\end{array}$ & $\begin{array}{l}\text { Emergency c-section vs } \\
\text { elective c-section } \\
\text { Adjusted odds ratio } \\
\text { (95\% Confidence interval) }\end{array}$ & $\begin{array}{l}\text { Vaginal delivery vs } \\
\text { elective c-section } \\
\text { Adjusted odds ratio } \\
\text { (95\% Confidence interval) }\end{array}$ \\
\hline \multicolumn{5}{|l|}{ Gender } \\
\hline Female & $2229(48.3)$ & 1.0 & 1.0 & 1.0 \\
\hline Male & $2386(51.7)$ & $1.23(1.03-1.45)^{*}$ & $1.04(0.82-1.33)$ & $0.85(0.67-1.09)$ \\
\hline \multicolumn{5}{|l|}{ Gestational age } \\
\hline 37 weeks and above & $3678(79.7)$ & 1.0 & 1.0 & 1.0 \\
\hline Less than 37 weeks & $937(20.3)$ & $0.86(0.69-1.07)^{*}$ & $0.72(0.53-0.99)^{*}$ & $0.85(0.62-1.14)$ \\
\hline \multicolumn{5}{|l|}{ Birthweight } \\
\hline $2500 \mathrm{~g}$ and above & $4044(87.6)$ & 1.0 & 1.0 & 1.0 \\
\hline Less than $2500 \mathrm{~g}$ & $571(12.4)$ & $0.79(0.60-1.03)$ & $1.36(0.88-2.10)$ & $1.73(1.12-2.67)^{*}$ \\
\hline \multicolumn{5}{|c|}{ Apgar score at I minute [a] } \\
\hline $0-6$ & $3956(90.4)$ & $1.15(0.84-1.56)$ & $0.82(0.53-1.27)$ & $0.72(0.47-1.09)$ \\
\hline $7-10$ & $419(9.6)$ & 1.0 & 1.0 & 1.0 \\
\hline \multicolumn{5}{|c|}{ Apgar score at 5 minutes [b] } \\
\hline $0-6$ & $1244(28.4)$ & $1.73(1.42-2.11)^{* * *}$ & $1.69(1.27-2.26)^{*}$ & $0.98(0.73-1.32)$ \\
\hline $7-10$ & $3131(71.6)$ & 1.0 & 1.0 & 1.0 \\
\hline \multicolumn{5}{|l|}{ Hyperbilirubinaemia } \\
\hline No & $4423(95.8)$ & 1.0 & 1.0 & 1.0 \\
\hline Yes & $192(4.2)$ & $1.39(0.90-2.16)$ & $0.42(0.24-0.74)^{*}$ & $0.30(0.17-0.55)^{* * * *}$ \\
\hline \multicolumn{5}{|l|}{ Admission into SCBU } \\
\hline No & $4148(89.9)$ & 1.0 & 1.0 & 1.0 \\
\hline Yes & $467(10.1)$ & $1.56(1.16-2.11)^{* *}$ & $2.19(1.30-3.70)$ & $\mathrm{I} .40(0.82-2.4 \mathrm{I})$ \\
\hline \multicolumn{5}{|l|}{ Hearing screening tests } \\
\hline Pass & $4296(93.1)$ & 1.0 & 1.0 & 1.0 \\
\hline Refer & $119(2.6)$ & $0.77(0.46-1.32)$ & $3.74(1.10-12.72)^{*}$ & $4.83(1.42-16.46)^{*}$ \\
\hline Incomplete & $200(4.3)$ & $0.80(0.52-1.22)$ & $1.53(0.73-3.20)$ & $1.92(0.92-4.00)$ \\
\hline
\end{tabular}

Missing data: [a], [b] = 240 (5.2\%); *p < 0.05; ** $\mathrm{p}<0.01$; *** $\mathrm{p}<0.001$;

$\$$ After adjusting for all maternal factors as well as all covariates. SCBU = special care baby unit; C-section = caesarean section; vs = versus $/$ compared with 
access to several private and public hospitals [23]. About $57 \%$ were attended by skilled health personnel suggesting that a few mothers who delivered outside hospital were still attended by skilled health personnel. Traditional maternity homes accounted for the largest proportion ( $40 \%$ ) of all deliveries or $77.5 \%$ of all non-hospital deliveries [23]. Our hospital is the only tertiary maternity hospital serving this population and possibly reflects the settings in many countries in sub-Saharan Africa. Evidently, maternal health-seeking behaviour in this urban setting is likely to reflect a complex interaction between socio-demographic, cultural and medical factors similar in some ways to those of mothers in rural communities $[24,25]$.

The observed caesarean section rate in this study is comparable to other local studies [26] but higher than the reported rates from other public health institutions which are usually in the range of 5\% to $43 \%$ where rates closer to the upper end are more typical in Latin America $[9,14,27]$. This high rate of caesarean section is not an indication of an indiscriminate preference by health professionals or mothers as has been observed in some developing countries [14,27] but of the referral status of the hospital in a community where majority of mothers prefer non-facility based delivery with a high probability of late presentation requiring surgical intervention during labour. Non-vaginal delivery is generally viewed as a sign of maternal laziness, reproductive failure or a curse from perceived enemies or deity in this population. It was therefore not uncommon even where caesarean section was indicated by past pregnancy history for women to attempt vaginal delivery until there was a glaring failure with obvious threat to the life of the mother or unborn child [28]. Qualitative studies have in fact established that some women will not even accept caesarean section under any circumstances for reasons such as the fear of pain or death, financial cost, embarrassment by friends, religious beliefs and husband's disapproval [13,25]. The delays associated with these and other factors $[8,12,29]$ may have contributed to the high proportion of emergency caesarean section (more than three-quarters of all caesarean sections). It may be worthwhile to undertake an audit to establish the relative contributions of the various types of delays to the high rate of emergency caesarean section and cases of near-misses in this setting.

Undoubtedly, a major pathway to the high rate of emergency caesarean section in this population was the lack of antenatal care which was associated with more than twofold risk compared to those who received antenatal care. Over half of the mothers who delivered by emergency caesarean section had no antenatal care. It was also not uncommon for some mothers who had received antenatal care to end-up with emergency caesarean section if they were culturally aversed to the potential for surgical inter- vention and had unsuccessfully attempted vaginal delivery within or outside a hospital setting [13,30,31]. Lack of antenatal care therefore remains a vital link between socio-demographic or obstetric risk factors and adverse pregnancy outcomes and perhaps the most modifiable of all risk factors [32].

Majority of the socio-demographic and obstetric factors associated with mode of delivery in this study accord with findings from studies in both developing and developed countries $[9,14,20,21]$. Factors less commonly reported are type of residential accommodation, sanitation facilities and use of herbal drug in pregnancy. These may be worth exploring more appropriately through future qualitative studies especially the use of herbal drug in pregnancy which perhaps mirrors the trend in both developed and developing countries towards the combined use of alternative/complimentary medicine and orthodox medicine to prevent adverse health outcomes or maximise treatment benefits [33].

The risks associated with obstetric complications and different modes of delivery are more commonly measured in terms of maternal and perinatal mortality [3], and this practice tends to underestimate the overall burden of the adverse health outcomes [34]. The current study provides further evidence that mode of delivery is associated with the risk of sensorineural hearing loss and complements our earlier report in which emergency caesarean section and vaginal delivery were associated with at least two-fold risk of sensorineural hearing loss in surviving newborns compared with elective caesarean section [17]. Our findings also suggest that while all caesarean sections combined may portend lesser risk for sensorineural hearing loss compared with vaginal delivery as reported by studies from countries with higher standards of obstetric practice $[35,36]$, infants delivered by emergency caesarean section were likely to be at a greater risk than those delivered by elective caesarean section in this resource-poor setting. It is in fact common in our population for emergency caesarean section to be initiated after prolonged and unsuccessful trial of labour due to several factors including cultural aversion to surgical intervention and financial constraints. In contrast, a related study among mothers with previous caesarean section found no association between mode of delivery and sensorineural hearing loss [28] which leads us to speculate that the incidence of fetal distress and the associated risks are less likely to be pronounced in pregnancies already considered as high risk as trial of labour in this group of women is likely to be more closely monitored for caesarean section than those without such obstetric indications. Although further confirmatory tests could not be provided for the majority of infants who failed the screening tests due to a high rate of follow-up default, our two-stage screening protocol typically has a test sensitivity of $92 \%$, specificity of $98 \%$ and 
positive likelihood ratio of 61 in hospital-based settings [37] and is currently the protocol of choice in many UNHS programmes worldwide.

Birth asphyxia (as indexed by low five-minute Apgar scores), hyperbirubinaemia and SCBU admission are established risk factors for a broad range of developmental disabilities such as cerebral palsy, mental retardation and specific language/attention deficit disorders [38], and the observed increased risks accord with existing extensive data on the respiratory morbidity associated with mode of delivery [26,39-41]. Other studies have also found that caesarean section is associated with adverse long-term health of survivors through reduced rates of breastfeeding [42]. While the increased risk of hyperbilirubinaemia associated with elective caesarean section warrants further investigation, the overall evidence from the current study would nonetheless suggest that the benchmark for evaluating the effectiveness of any mode of delivery should not be limited to the number of deaths averted but also the risks of developmental disabilities in surviving newborns particularly when emergency caesarean section is necessitated in already compromised infants. Mothers and health care providers need to appreciate this added dimension to obstetric care in developing countries. The association between infant's gender and mode of delivery was an unusual finding that needs to be explored further in future studies.

Major advantages of this study include the comparative analysis of three modes of delivery simultaneously together with the associated neonatal outcomes seldom found in similar studies from developing countries. This study has also demonstrated that newborn screening programmes offer unique platforms for establishing interrelationships among various determinants of maternal and neonatal outcomes besides the benefits of early detection for timely intervention. However, a number of limitations of this retrospective cross-sectional study are worth noting. It is uncertain how the findings in this study can be generalised for other tertiary health institutions within different cultural settings outside sub-Saharan Africa in view of the selection bias. Adverse outcomes considered in this study excluded maternal and perinatal mortality associated with the obstetric practices but this burden is already well documented in existing literature. The potential impact of the cost of caesarean section on maternal decision was not ascertained although this was less likely to be a major factor in this state-funded public hospital compared with fees charged by private hospitals. Data on the number of antenatal visits made or the number of previous caesarean sections among those with this history was also not considered. Prospective studies addressing these limitations as well as exploring the interaction effects of parity are necessary to support the findings in this study [19]. Analysis of time interval between admis- sion and caesarean section (to indicate degree of urgency) and analysis of subsequent length of hospital stay (to indicate severity of outcome) as well as a study of the long-term outcomes for these "near-miss" obstetric events would be valuable.

\section{Conclusion}

This study has shown that the rate of emergency caesarean section in a tertiary referral hospital is likely to be high in a community where a high proportion of women prefer to deliver outside hospitals. This pattern of delivery is associated with several obstetric complications and adverse neonatal outcomes which portend substantial risks of developmental deficits such as sensorineural hearing loss in the surviving newborns. Efforts aimed at improving maternal and child mortality in this and similar settings must recognise the broader dimensions of the burden of obstetric complications associated with emergency caesarean section.

\section{Abbreviations}

C-Section: caesarean section; SCBU: special care baby unit; UNHS: universal newborn hearing screening.

\section{Competing interests}

The authors declare that they have no competing interests.

\section{Authors' contributions}

BOO designed the study protocol as part of a wider postdoctoral research project. OAS participated in the data collection, contributed to data analysis and interpretation. BOO drafted the manuscript with inputs from OAS. Both authors reviewed and approved the final manuscript. $\mathrm{BOO}$ is guarantor of the paper.

\section{Acknowledgements}

The instruments for the substantive newborn hearing screening project that formed the basis of this work were provided by Natus Medical Inc., USA, Otodynamics (UK) Ltd and Oticon Foundation, Denmark. Training support was received from NHS Newborn Hearing Screening Programme/ MRC Hearing and Communication Group, UK. Educational materials for parents and health professionals were sponsored by the Education Trust Fund (ETF) an agency of the Federal Government of Nigeria. Hearing International Nigeria (HING), a local charity provided financial support for all the operational costs of the project. None of the sponsors was involved in the study design, collection, analysis and interpretation of data; the writing of the manuscript; or the decision to submit these results for publication.

\section{References}

I. Kerber KJ, Graft-Johnson JE, Bhutta ZA, Okong P, Starrs A, Lawn JE: Continuum of care for maternal, newborn, and child health: from slogan to service delivery. Lancet 2007, 370: I358-I 369.

2. World Health Organisation, World Bank, UNICEF, United Nations Population Fund: Maternal mortality in 2005: estimates developed by WHO, UNICEF, UNFPA, and the World Bank. Geneva. World Health Organisation; 2007.

3. Ronsmans C, Graham WJ, Lancet Maternal Survival Series steering group: Maternal mortality: who, when, where, and why. Lancet 2006, 368: I|89-1200. 
4. Black RE, Morris SS, Bruce J: Where and why are $\mathbf{1 0}$ million children dying every year? Lancet 2003, 361:2226-2234.

5. Lawn JE, Cousens S, Zupan J: 4 million neonatal deaths: When? Where? Why? Lancet 2005, 365:891-900.

6. Campbell OM, Graham WJ, Lancet Maternal Survival Series steering group: Strategies for reducing maternal mortality: getting on with what works. Lancet 2006, 368: I284-I 299.

7. United Nations: The millennium development goals report 2008. New York. United Nations; 2008.

8. Thaddeus S, Maine D: Too far to walk: maternal mortality in context. Soc Sci Med 1994, 38: 109I-IIIO.

9. Kwawukume EY: Caesarean section in developing countries. Best Pract Res Clin Obstet Gynaecol 200I, 15:165- I78.

10. Grossmann-Kendall F, Filippi V, De Koninck M, Kanhonou L: Giving birth in maternity hospitals in Benin: testimonies of women. Reprod Health Matters 200I, 9:90-98.

II. Chigbu CO, lloabachie GC: The burden of caesarean section refusal in a developing country setting. BJOG 2007, I 14:1261-1265.

12. Koblinsky M, Matthews Z, Hussein J, Mavalankar D, Mridha MK, Anwar I, Achadi E, Adjei S, Padmanabhan P, Marchal B, De Brouwere V, van Lerberghe W, Lancet Maternal Survival Series steering group: Going to scale with professional skilled care. Lancet 2006 368: $1377-1386$.

13. Aziken M, Omo-Aghoja L, Okonofua F: Perceptions and attitudes of pregnant women towards caesarean section in urban Nigeria. Acta Obstet Gynecol Scand 2007, 86:42-47.

14. Villar J, Valladares E, Wojdyla D, Zavaleta N, Carroli G, Velazco A, Shah A, WHO 2005 global survey on maternal and perinatal health research group, et al.: Caesarean delivery rates and pregnancy outcomes: the 2005 WHO global survey on maternal and perinatal health in Latin America. Lancet 2006, 367:1819-1829.

15. Filippi V, Ganaba R, Baggaley RF, Marshall T, Storeng KT, Sombié I, Ouattara F, Ouedraogo T, Akoum M, Meda N: Health of women after severe obstetric complications in Burkina Faso: a longitudinal study. Lancet 2007, 370:1329-1337.

16. Ashford $L$ : Hidden suffering: disabilities from pregnancy and childbirth in less developed countries. Policy Brief: Population Reference Bureau. Washington DC; 2002.

17. Olusanya BO: Newborns at risk of sensorineural hearing loss in low-income countries. Arch Dis Child 2009, 94:227-230.

18. Grantham-McGregor S, Cheung YB, Cueto S, Glewwe P, Richter L, Strupp B, International Child Development Steering Group: Developmental potential in the first 5 years for children in developing countries. Lancet 2007, 369:60-70.

19. Khan KS, Wojdyla D, Say L, Gülmezoglu AM, Van Look PF: WHO analysis of causes of maternal death: a systematic review. Lancet 2006, 367:1066-1074.

20. Patel RR, Peters TJ, Murphy DJ, ALSPAC Study Team: Prenatal risk factors for Caesarean section. Analyses of the ALSPAC cohort of 12,944 women in England. Int J Epidemiol 2005, 34:353-367.

21. Dumont A, de Bernis L, Bouvier-Colle MH, Bréart G, MOMA study group: Caesarean section rate for maternal indication in subSaharan Africa: a systematic review. Lancet 200I, 358: $1328-1333$.

22. Olusanya $O$, Okpere $E$, Ezimokhai M: The importance of social class in voluntary fertility. West Afr J Med 1985, 4:205-2 I 2 .

23. Olusanya BO, Ebuehi OM, Somefun AO: Universal infant hearing screening programme in a community with predominant non-hospital births: a three-year experience. J Epidemiol Comm Health 2009, 63:48I-486.

24. Izugbara CO, Afangideh Al: Urban women's use of rural-based health care services: the case of Igbo women in Aba City, Nigeria. J Urban Health 2005, 82:III-I2I.

25. Onah HE, Ikeako LC, lloabachie GC: Factors associated with the use of maternity services in Enugu, southeastern Nigeria. Soc Sci Med 2006, 63:1870-1878.

26. Njokanma FO, Egri-Okwarji MTC, Nwokoro CA, Orebamjo T, Okeke GCE: Birth asphyxia, perinatal and maternal mortality associated with caesarean section. Trop J Obstet Gynaecol 2002, 19:25-29.

27. Ronsmans C, Holtz S, Stanton C: Socioeconomic differentials in caesarean rates in developing countries: a retrospective analysis. Lancet 2006, 368:1516-1523.
28. Olusanya BO, Solanke OA: Adverse neonatal outcomes associated with previous cesarean section in an inner-city maternity hospital in Lagos, Nigeria. Inter J Gynaecol Obstet 2009. [Epub ahead of print]

29. Filippi V, Richard F, Lange I, Ouattara F: Identifying barriers from home to the appropriate hospital through near-miss audits in developing countries. Best Pract Res Clin Obstet Gynaecol 2009, 23:389-400

30. Etuk SJ, Itam IH, Asuquo EE: Morbidity and mortality in booked women who deliver outside orthodox health facilities in Calabar, Nigeria. Acta Trop 2000, 75:309-313.

3I. Etuk SJ, Etuk IS, Ekott MI, Udoma E): Perinatal outcome in pregnancies booked for antenatal care but delivered outside health facilities in Calabar, Nigeria. Acta Trop 2000, 75:29-33.

32. Magadi M, Diamond I, Madise N, Smith P: Pathways of the determinants of unfavourable birth outcomes in Kenya. J Biosoc Sci 2004, 36: I53-176.

33. Astin JA: Why patients use alternative medicine: results of a national study. JAMA 1998, 279: I548-1553.

34. Storeng KT, Baggaley RF, Ganaba R, Ouattara F, Akoum MS, Filippi V: Paying the price: the cost and consequences of emergency obstetric care in Burkina Faso. Soc Sci Med 2008, 66:545-557.

35. Bener A, Eihakeem AA, Abdulhadi $\mathrm{K}$ : Is there any association between consanguinity and hearing loss. Int J Pediatr Otorhinolaryngol 2005, 69:327-333.

36. Thiringer K, Kankkunen A, Lidén G, Niklasson A: Perinatal risk factors in the aetiology of hearing loss in preschool children. Dev Med Child Neurol 1984, 26:799-807.

37. Kennedy C, McCann D, Campbell MJ, Kimm L, Thornton R: Universal newborn screening for permanent childhood hearing impairment: an 8-year follow-up of a controlled trial. Lancet 2005, 366:660-662.

38. Committee on Nervous System Disorders in Developing Countries, Board on Global Health: Neurological, psychiatric and developmental disorders: meeting the challenge in the developing world. Washington D.C. National Academy Press; 200 I.

39. Liston FA, Allen VM, O'Connell CM, Jangaard KA: Neonatal outcomes with caesarean delivery at term. Arch Dis Child Fetal Neonatal Ed 2008, 93:FI76-182.

40. Levine EM, Ghai V, Barton J], Strom CM: Mode of delivery and risk of respiratory diseases in newborns. Obstet Gynecol 200I, 97:439-442.

4I. Landon MB, Hauth JC, Leveno KJ, Spong CY, Leindecker S, Varner $\mathrm{MW}$, Moawad AH, et al.: Maternal and perinatal outcomes associated with a trial of labor after prior cesarean delivery. $N$ Engl J Med 2004, 35 I:258I-2589.

42. Rowe-Murray HJ, Fisher JRW: Baby friendly hospital practices: caesarean section is a persistent barrier to early initiation of breastfeeding. Birth 2002, 29:|24-|3|.

\section{Pre-publication history}

The pre-publication history for this paper can be accessed here:

\section{http://www.biomedcentral.com/1471-2393/9/41/prepub}

Publish with BioMed Central and every scientist can read your work free of charge

"BioMed Central will be the most significant development for disseminating the results of biomedical research in our lifetime. "

Sir Paul Nurse, Cancer Research UK

Your research papers will be:

- available free of charge to the entire biomedical community

- peer reviewed and published immediately upon acceptance

- cited in PubMed and archived on PubMed Central

- yours - you keep the copyright 